\title{
Co-administration effects of a-lipoic acid and nucleo CMP on arousal and sensory cortical activity
}

\author{
Hayder M. Al-kuraishy ${ }^{1}$ and Ali I. Al-Gareeb ${ }^{2}$ \\ Department of Pharmacology, Toxicology and Medicine College of Medicine Almustansiriya University, Baghdad, Iraq.
}

\begin{abstract}
Objective: To evaluate the combined effects of lipoic acid and nuclear CMP on arousal and sensory cortical activity in normal healthy volunteers. Method: Eighty healthy volunteers enrolled in this study, the volunteers were divided into four groups'. Group A: take a placebo and regarded as controlling. Group B: take nucleo CMP capsule $5 \mathrm{mg} /$ day. Group C: take lipoic acid $400 \mathrm{mg} /$ day. Group D: take nucleo CMP and lipoic acid/ day. The duration of treatment was two weeks. CFFF tests measured via Leeds psychomotor battery tester. Student t-test, simple correlation and ANOVA test via SPSS version 18 , taking $p<0.05$ as the lowest limit of significance. Results: lipoic acid improves fusion frequency (ascending) insignificantly and flicker (descending) frequency significantly in normal healthy volunteers. Nucleo CMP leads to a significant amelioration of critical flicker frequency, flicker percent and fusion percent $p<0.05$, but, it produced insignificant effects on a flicker index, critical fusion frequency and fusion index $p>0.05$. Combined lipoic acid and Nucleo CMP produced significant effects on critical flicker frequency, critical fusion frequency, flicker percent and fusion percent $p<0.05$, but they produced insignificant effects on a flicker index, fusion index, and CFFF $p>0.05$. Conclusion: lipoic acid and nucleo CMP produced significant arousal effects on normal healthy volunteers and improve cognitive functions.

Key-words: CFFF, Lipoic acid, Nucleo CMP, Sensory cortical activity
\end{abstract}

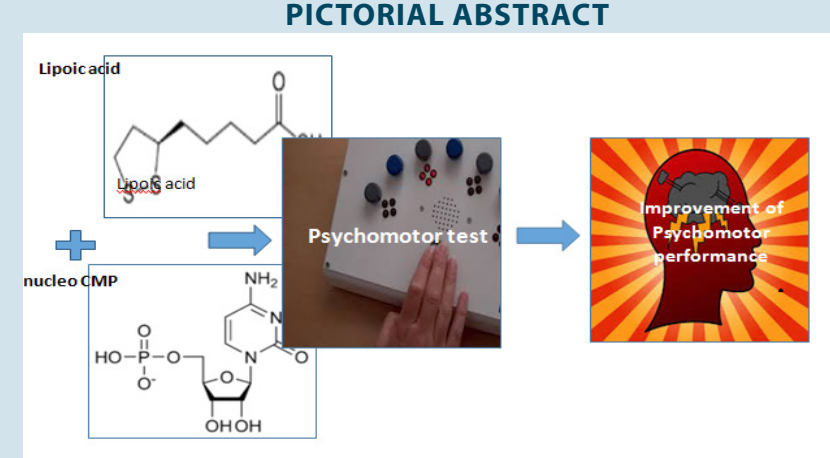

Correspondence :

Hayder M. Al-kuraishy

College of Medicine Almustansiriya University

P.O. Box 14132 Iraq /Baghdad

Phone numbers Tel. +96407906230487

E-mail: Hayderm36@Yahoo.Com

DOI : 10.5530/jyp.2016.1.4

\section{INTRODUCTION}

The critical fusion flicker frequency (CFFF), measures the frequency threshold of light perception of flickering to fusion and vice versa, via visual processing system, thus; CFFF, regarded as the lowest tempo of unremitting flicker that perceived steady light. ${ }^{1}$ Thalamic and retinal neurons are reacting to flicker rate above $100 \mathrm{~Hz}$ and, CFFF values were low in frontal lesions and with using of sedative drugs, and there is a strong correlation between intelligence and CFFF. ${ }^{2}$

Normally human brains detects light flickering at definite frequency, when this frequency elevated it called the threshold and flickering light appeared continuous (fusion) this called CFFF threshold due to loss of discriminating ability for differentiation between flicker and fusion light because the flicker become invisible, but when the rate of frequency decreased from sub-threshold areas the flickering light appeared again ,moreover, CFFF measures cortical arousal and considered as an index for discrete sensory actions it detects fatigue and psychomotor retardation, but there is some controversy about the validity of CFFF normal human CFFF ranged from $36-39 \mathrm{~Hz}$, which was dependent on measurement methods and deceiving by psychophysical and electrophysiological measurements. ${ }^{3,4}$

Therefore, modulation of visual cortex experience affects the CFFF; as a result, lesions in magnocellular pathway or occipital cortex leading to prolongation of CFFF, these indicating the cortical origin of CFFF that reflect on a brain cortical function. ${ }^{5}$

In animal model studies, CFFF considered as a scientific tool for measurement of cortical processing, also; motion direction performance im- provement with increasing neuronal sensitivity for sub- threshold plasticity and visual discrimination. ${ }^{6}$

CFFF is highly sensitive to intrinsic and extrinsic factors, and affected by numerous factors like target size, colour, luminance, retinal diseases, gender, person age (it decreases with age), stress and other extrinsic factors like time and environmental noise. Thus, any results obtained should indicate the impact on extrinsic and intrinsic factors, additionally, CFFF measure cortical processing capability and capacity, thus, it regarded as a diagnostic measure for different neurological diseases likes multiple sclerosis, schizophrenia, Alzheimer dementia also, CFFF used as a diagnostic tool in the psychopharmacological work for detection of cognitive adverse effects of sedative and suppressant drugs. ${ }^{7}$

The human brain is highly sensitive to free radicals, damaging due to high oxygen consumption, high metabolism and large phospholipids' content which leading to cognitive dysfunction, worsening of neurotransmitter metabolism and the reduce cortical processing capacity all these may deteriorate CFFF, therefore, drugs like lipoic acid or nucleo CMP like other herbal medicine may affect CFFF that reflect the cortical arousal state. ${ }^{8,9}$

a-lipoic acid is a natural antioxidant found in all human cells, but in very small amount, it is hydro and lipophilic, protect DNA from oxidative damage also, improves neuronal blood flow, augment nerve transmission and prevent neuronal injury to acute inflammation. ${ }^{10}$ Moreover, it regenerates and preserves vitamin $\mathrm{E}$ and $\mathrm{C}$, protect coenzyme Q10 and glutathione leading to powerful antioxidant effects, it is also; energetically because it's the first enzyme that converted glucose to ATP, leading to energy production, and regarded as a determiner for mitochondrial 
metabolic rate ,also, it protects neuronal membranes and mitochondrial DNA specifically from free radical formation during oxidative stress ,it also acts as a shelter for iron, so; prevent progressions of iron-dependent oxidative damage. ${ }^{11}$

Nucleotide based drugs like nucleocytidine monophosphate CMP mainly used for peripheral neurological disorders like trigeminal neuralgia, diabetic neuropathy and lumbosciaticalgia , but it central roles remains to be elucidated, Nucleo CMP improve neural growth and never repair, improvement, regeneration of myelinated nerve fibre, delay spinal pain transmission, enhance spinal density and acceleration of hippocampal dependent working memory in animal model study also; Nucleo CMP contain uridine monophosphate, uridine diphosphate and uridine triphosphate, which together with cysteine monophosphate induce biosynthesis of neuronal glycolipid, phospholipid, RNA and DNA, Nucleo CMP crosses the blood brain barrier and then phosphorylated into uridine triphosphate that lead to triggering of neurotransmitter modulation. ${ }^{12}$

Therefore, the aim of the present study was the evaluation of combined effects of lipoic acid and nuclear CMP on arousal and sensory cortical activity that is considering through the estimation of CFFF in normal healthy volunteers.

\section{SUBJECTS AND METHODS}

Eighty healthy volunteers (55 males and 25 females) enrolled in this study aged between 22-23years. All volunteers were trained as beginning the experiment at comfortable condition, at the research unit in Department of Clinical Pharmacology, College of Medicine, Al-Mustansiriyia University, Iraq -Baghdad during January 2015.

\section{Study design}

The volunteers were dividing into four groups:

Group A: take a placebo (500mg starch) which regarded as controlling. Group B: take nucleo CMP capsule orally $5 \mathrm{mg} /$ day.

Group C: take lipoic acid tablet orally $400 \mathrm{mg} /$ day

Group D: take both nucleo CMP and lipoic acid/ day

The duration of treatment was two weeks.

\section{Measurement of critical-flicker fusion frequency (CFFF). ${ }^{13}$}

CFFF test was measuring via Leeds psychomotor battery tester ( $\mathrm{Zac}$ Gmbh.D-8346-Simbach/Inn). The test done at dimes timed at 9a.m, the device contained four red emitting diodes placed into a center of a $20 \mathrm{~mm}$ squared above blank board. The volunteer is supposed to sit in facing of tester about $75 \mathrm{~cm}$ distances from an eye to the tester to ensuring binocular vision. The flicker frequency started as constant and steady red light, once it flickering the volunteer should press the button, this called descending frequency, and it ranged from $1-30 \mathrm{~Hz}$. The fusion frequency started as flickering red light, once it became steady or constant this called ascending frequency, it ranged from 30-60 Hz.

The tester device recorded and calculated four fusions and four flickering then, mean of those was taken, and the average of critical fusion flickering frequency CFFF representing arousal and sensory cortical activity. Then from above flickering and fusion, we can calculate the following Flicker index $=\max / \max +\min (\operatorname{maximum}$ and minimum values in critical flicker frequency )

Percent flicker $=\max -\min / \max +\min 100$ (maximum and minimum values in critical flicker frequency)

Fusion index $=\max / \max +\min ($ maximum and minimum values in critical fusion frequency )

Percent fusion $=\max -\min / \max +\min 100($ maximum and minimum val ues in critical fusion frequency)
$\mathrm{CFFF}=$ flicker frequency+fusion frequency $/ 2$

\section{Statistical analysis}

The results presented as mean $\pm \mathrm{SE}$, they were analyses through using the student t-test, simple correlation and ANOVA test via SPSS version 18, taking $\mathrm{p}<0.05$ as the lowest limit of significance.

\section{RESULTS}

Results are presented for 88 volunteers $(62.5 \%$ males and $28.40 \%$ females) with 8 (9.090\%) was withdrawn from this study, as well as 80 volunteers $(90 \%)$ completes this study Figure (1).

Placebo effects produced insignificant effects on all parameters of flickerfusion frequency $\mathrm{p}>0.05$, while lipoic acid produced significant effects on critical flicker frequency, flicker and fusion percent $p<0.05$, except CFFF, fusion index and flicker index where lipoic acid produced insignificant $\mathrm{p}>0.05$.

Critical flicker frequency reduced from $30.38 \pm .304 \mathrm{~ms}$ to $28.72 \pm .235$ $\mathrm{ms}$, while critical fusion frequency increased from $32.03 \pm .359 \mathrm{~ms}$ to $33.347 \pm .376 \mathrm{~ms}$ as in comparison the effects of lipoic acid with control, table (1). Therefore, lipoic acid improves fusion frequency (ascending) insignificantly and flicker (descending) frequency significantly in normal healthy volunteers.

Table 1: Effect of lipoic acid on flicker-fusion frequency parameters in comparison with control.

\begin{tabular}{cccc}
\hline Flicker-fusion variables & Control $(\mathbf{n}=\mathbf{2 0})$ & $\mathrm{L} . \mathrm{A}(\mathbf{n}=\mathbf{2 0})$ & $p$ \\
$\begin{array}{c}\text { Critical flicker } \\
\text { frequency(Hz) }\end{array}$ & $30.38 \pm .304$ & $28.72 \pm .235$ & $0.006^{*}$ \\
Flicker index(Hz) & $.501 \pm .0003$ & $.501 \pm .0006$ & 0.22 \\
Flicker percent (\%) & $.370 \pm .066$ & $.226 \pm .040$ & $0.0001^{*}$ \\
$\begin{array}{c}\text { Critical fusion } \\
\text { frequency(Hz) }\end{array}$ & $32.03 \pm .359$ & $33.347 \pm .376$ & 0.09 \\
Fusion index(Hz) & $.501 \pm .0002$ & $.5009 \pm .0004$ & 0.248 \\
Fusion percent (\%) & $.318 \pm .0555$ & $.185 \pm .0284$ & $0.0001^{*}$ \\
CFFF(Hz) & $31.20 \pm .211$ & $31.03 \pm .207$ & 0.75 \\
\hline
\end{tabular}

${ }^{*} p$ significant effect $<0.05, \mathrm{~L} . \mathrm{A}=$ lipoic acid, $\mathrm{CFFF}=$ critical flicker fusion frequency.

Nucleo CMP lead to significant ameliorations of critical flicker frequency, Flicker percent (\%) and fusion percent $\mathrm{p}<0.05$, but, it produced insignificant effects on flicker index , critical fusion frequency and Fusion index $\mathrm{p}>0.05$ Table 2 .

Combined lipoic acid and Nucleo CMP produced significant effects on critical flicker frequency, Critical fusion frequency, Flicker percent and

Table 2: Effects of Nucleo CMP on flicker-fusion frequency parameters in comparison with control.

\begin{tabular}{cccc}
\hline Flicker-fusion variables & Control $(\mathbf{n}=\mathbf{2 0})$ & $\mathrm{N} . \mathrm{C}(\mathbf{n}=\mathbf{2 0})$ & $p$ \\
$\begin{array}{c}\text { Critical flicker } \\
\text { frequency(Hz) }\end{array}$ & $30.38 \pm .304$ & $29.25 \pm .318$ & $0.02^{*}$ \\
Flicker index(Hz) & $.501 \pm .0003$ & $.500 \pm .0000092$ & 0.13 \\
Flicker percent (\%) & $.370 \pm .066$ & $.170 \pm .001$ & $0.00002^{*}$ \\
Critical fusion & $32.03 \pm .359$ & $32.76 \pm .234$ & 0.633 \\
frequency(Hz) & & & \\
Fusion index(Hz) & $.501 \pm .0002$ & $.5006 \pm .00009$ & 0.245 \\
Fusion percent $(\%)$ & $.318 \pm .0555$ & $.125 \pm .0183$ & $0.00001^{*}$ \\
CFFF(Hz) & $31.20 \pm .211$ & $31.01 \pm .168$ & 0.66 \\
\hline
\end{tabular}

${ }^{*} p$ significant effect $<0.05, \mathrm{~N} . \mathrm{C}=$ Nucleo $\mathrm{CMP}, \mathrm{CFFF}=$ critical flicker fusion frequency . 
CONSORT diagram showing the flow of participants through each stage of the present study.

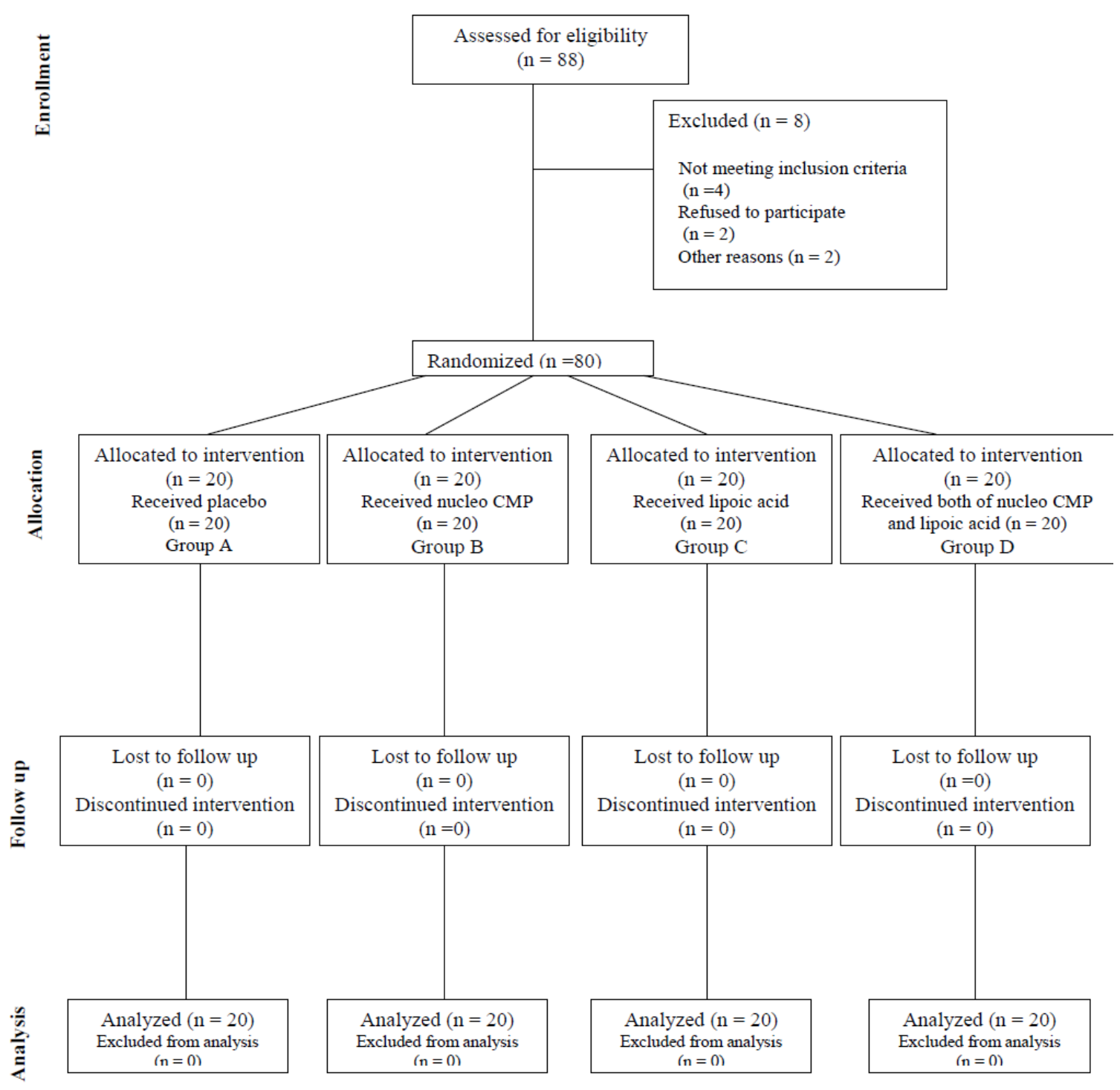

Figure 1: subject flow diagram

Table 3: Combined effect of lipoic acid and Nucleo CMP on flicker-fusion frequency parameters in comparison with control.

\begin{tabular}{cccc}
\hline $\begin{array}{c}\text { Flicker-fusion } \\
\text { variables }\end{array}$ & Control $(\mathbf{n = 2 0 )}$ & L.A+ N.C $(\mathbf{n = 2 0 )}$ & $p$ \\
$\begin{array}{c}\text { Critical flicker } \\
\text { frequency(Hz) }\end{array}$ & $30.38 \pm .304$ & $26.78 \pm .124$ & $0.00029^{*}$ \\
Flicker index(Hz) & $.501 \pm .0003$ & $.5008 \pm .00006$ & 0.17 \\
Flicker percent (\%) & $.370 \pm .066$ & $.173 \pm .013$ & $0.00001^{*}$ \\
Critical fusion & $32.03 \pm .359$ & $35.73 \pm .414$ & $0.009^{*}$ \\
frequency(Hz) & & & \\
Fusion index(Hz) & $.501 \pm .0002$ & $.500 \pm .00002$ & 0.257 \\
Fusion percent $(\%)$ & $.318 \pm .0555$ & $.145 \pm .005$ & $0.0002^{*}$ \\
CFFF(Hz) & $31.20 \pm .211$ & $31.26 \pm .208$ & 0.76 \\
\hline
\end{tabular}

${ }^{*} p$ significant effect $p<0.05, \mathrm{~L}+\mathrm{N}=\mathrm{Lipoic}+$ Nucleo CMP, CFFF=critical flicker fusion frequency

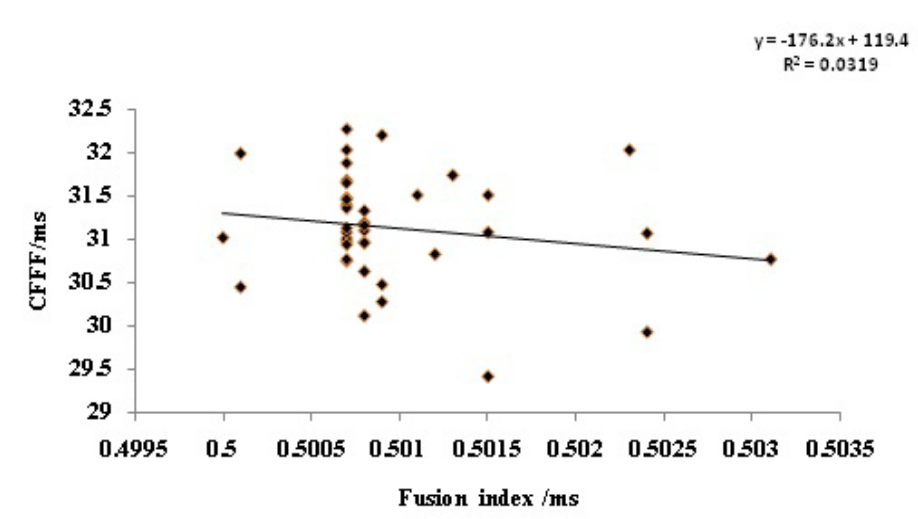

Figure 2: Correlation between fusion index and CFFF. 
Table 4: Intra and intergroup variations in critical flicker-fusion frequency parameters.

\begin{tabular}{|c|c|c|c|c|c|c|}
\hline Flicker-fusion variables & $\begin{array}{l}\text { Control } \\
(n=20)\end{array}$ & $\begin{array}{c}\text { N.C } \\
(n=20)\end{array}$ & $\begin{array}{c}\text { L.A } \\
(n=20)\end{array}$ & L.A+N.C $(n=20$ & $\mathrm{F}$ & Sig. \\
\hline Critical flicker frequency $(\mathrm{Hz})$ & $30.38 \pm .304$ & $29.25 \pm .318$ & $28.72 \pm .235$ & $26.78 \pm .124$ & 34.069 & .000 \\
\hline Flicker index $(\mathrm{Hz})$ & $.501 \pm .0003$ & $.500 \pm .0000092$ & $.501 \pm .0006$ & $.5008 \pm .00006$ & 5.642 & .003 \\
\hline Flicker percent (\%) & $.370 \pm .066$ & $.170 \pm .001$ & $.226 \pm .040$ & $.173 \pm .013$ & 5.642 & .003 \\
\hline Critical fusion frequency $(\mathrm{Hz})$ & $32.03 \pm .359$ & $32.76 \pm .234$ & $33.347 \pm .376$ & $35.73 \pm .414$ & 20.657 & .000 \\
\hline Fusion index $(\mathrm{Hz})$ & $.501 \pm .0002$ & $.5006 \pm .00009$ & $.5009 \pm .0004$ & $.500 \pm .00002$ & 7.072 & .001 \\
\hline Fusion percent (\%) & $.318 \pm .0555$ & $.125 \pm .0183$ & $.185 \pm .0284$ & $.145 \pm .005$ & 7.072 & .001 \\
\hline $\operatorname{CFFF}(\mathrm{Hz})$ & $31.20 \pm .211$ & $31.01 \pm .168$ & $31.03 \pm .207$ & $31.26 \pm .208$ & .390 & .761 \\
\hline
\end{tabular}

$P$ value calculating via ANOVA Bonferrony test

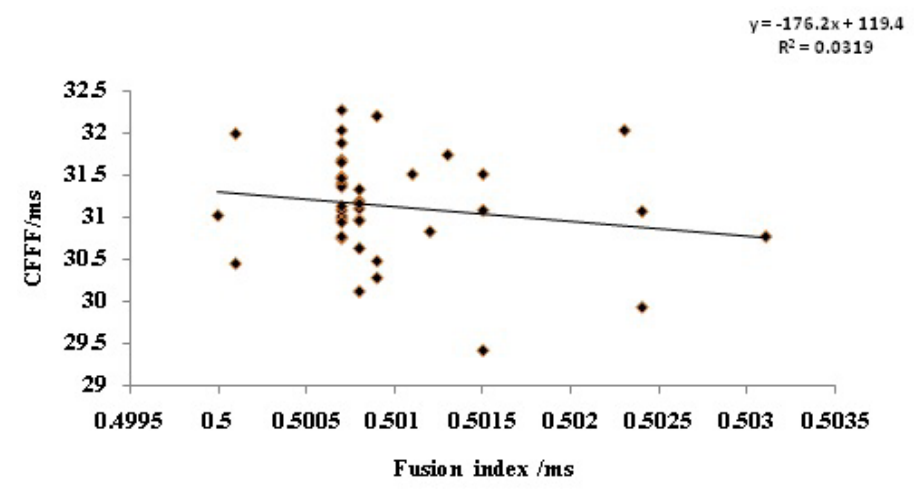

Figure 3: Correlation between flicker index and CFFF.

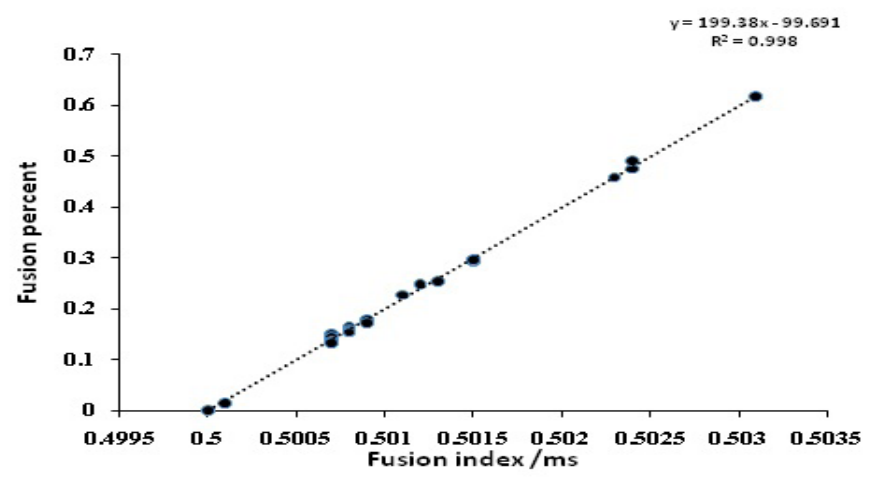

Figure 4: Correlation between fusion index and fusion percent.

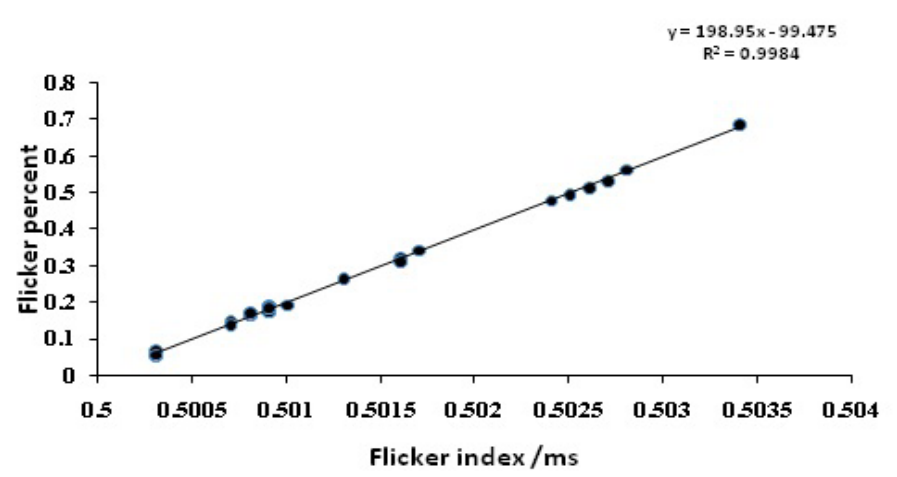

Figure 5: Correlation between flicker index and flicker percent.
Fusion percent $\mathrm{p}<0.05$, but they produced insignificant effects on a flicker index, Fusion index, and CFFF p $>0.05$ Table 3.

Therefore, neither lipoic acid nor Ncleo CMP ameliorate or improve the values of $\mathrm{CFFF}$ in comparison with control. Inter-group and intra-group variations shows significant effects in all parameters ( $p$ value ranged from 0.00001 to 0.03 except CFFF changes that was insignificant $p=0.761$ table (4).

Fusion index negatively correlated with CFFF while, it moderately correlated with CFFF in all groups Figure 2, 3.

Moreover, there is a significant positive correlation between fusion index and fusion percent $\mathrm{R}^{2}=0.998$, also, there is positive correlation regarding flicker index and flicker percent $\mathrm{R}^{2}=0.998$ Figure 4,5 .

\section{DISCUSSION}

This study suggests that lipoic acid and nucleo CMP produced significant arousal effect on normal healthy volunteers and improve cognitive functions as compared with placebo effects also; the present study shows that lipoic acid significantly improves critical flicker frequency, fusion index and percent fusion but insignificantly improve other parameters. Since, CFFF test is a reliable and simple test for evaluation of central arousal state that used in the present study to investigate different drugs that mainly acts peripherally or as antioxidant on cognitive arousal function. A Numerous studies ${ }^{14}$ showed the variability in CFFF estimation that regarded with the present study theses variability includes gender, age, race and health factors .

Critical flicker frequency measures cognitive function and regarded as indicator for sedative drugs index, while critical fusion frequency measure occipital cortex capacity, therefore, there are different cortical neuronal pathways to flickers and fusion frequency. ${ }^{15}$ lipoic acid, in the present study, improves critical flicker frequency so; it advancing the cognitive function.

Lipoic acid is a potent antioxidant, improve mitochondrial function, regulate redox condition and scavenging reactive oxygen species so, it terminates free radical effects, also ,it inhibits lipid peroxidation and reactivate endogenous antioxidant, these findings explained the neuroprotective effects of lipoic acid in the management of neuropathy as it is well known that oxidative stress regardless of it causes were implicated in neurodegenerative disorders. ${ }^{16}$

Additionally, lipoic acid produced cytoprotective effects in cerebral ischemic reperfusion injury via direct antioxidant effects or through activation of endogenous glutathione and/or elevation of vitamins $\mathrm{E}$ and C. ${ }^{17}$

A deficiency in cerebral glutathione leads to deterioration in cognitive function, spatial memory and psychomotor performance moreover; lipoic acid chelators lead to significant deterioration and declining in 
scores of spatial learning and spatial memory due to potent inhibition of hippocampal facilitation and long term potentiating. In addition, deficiency in lipoic acid causes cognitive deficiency and deterioration in cerebral retention capacity with little effects on sensory-motor motivational capability thus, long-term insufficiency in glutathione linked enzymes lead to structural and functional neuronal dys-connectivity and impairment of cognitive function, also; lipoic acid improves working memory in rats that treated by lipoic acid for two weeks duration via modulation of glutamate NMDA receptors. ${ }^{18}$

Vitamin C modulates brain neurotransmitter system mainly glutaminergic,dopaminergic, cholinergic and serotonergic that are linked in memory, learning and cognitive function, moreover, vitamin $\mathrm{C}$ regarded as important cofactor in the synthesis and releasing of noradrenaline and cerebral acetylcholine ${ }^{19}$,this may explained the cognitive enhancing effects of lipoic acid that augments endogenous cerebral ascorbate level in aged and healthy subjects also, neurobehavioral effect of different herbs was explained depending on their antioxidant properties.$^{20}$

Animal model study showed that mice deficient in gene encoding for vitamin $\mathrm{C}$ lactone oxidase developed a sever cognitive impairment due to oxidative stress that measured by F4-neuroprostans, also, vitamin $\mathrm{C}$ reverses and attenuate scopolamine induced cognitive impairment in rats due to stimulation of central acetylcholineastrase enzyme at median forebrain bundle or through activation of cholinergic neurons, and in spite of vitamin $\mathrm{C}$ does not cross blood brain barrier, ${ }^{21,22}$ therefore ,oral and intraperitoneal administration of vitamin $\mathrm{C}$ will not elevates cerebral ascorbate level ,but lipoic acid elevate brain ascorbate concentration that be able for central arousal effects which improve attention and learning due to modulation of hippocampal and forebrain glutamate concentration. Moreover, lipoic acid may improve psychomotor performance through its modulator effects on glutamate receptor which per see improve a brain nitric oxide. ${ }^{23}$

Therefore, lipoic acid acts as direct antioxidant or indirect via elevation of endogenous antioxidant and glutathione concentration, modulations of nitric oxide and glutamate, also it inhibits peroxynitrite induced neuronal damage, all these effects may explain the acceleration in critical flicker frequency in the present study which reflects arousal enhancing effects on normal healthy volunteers.

Additionally, the present study revealed that Nucleo CMP significantly improves critical flicker frequency, critical fusion frequency and fusion index, thus it improve both cognitive and visual perception functions of normal persons.

Nucleo CMP prevents cerebral ischemia and degenerative brain disease that related to the modulation of purinergic receptors. ${ }^{24}$

Purinergic receptors, are concerned with memory and learning functions, these receptor responsible for regulation of migration, apoptosis and proliferation of neuronal stem cells, which may be related to the activation of mitogen activated protein kinase (MAPK) pathway that lead to cellular proliferation via upregulation of purinergic receptor (P2Y. ${ }^{25}$ Nucleo CMP also leads to significant dopaminergic neuronal activation and neuroprotection via preservation of mitochondrial transition pore depolarization from ischemic hypoxic neuronal injury and prevention of glutamate-induced neurotoxicity. ${ }^{26}$ Therefore, nucleo CMP produced significant cognitive and arousal enhancement effects due to activation of purinergic and dopaminergic receptors or/and modulation of glutaminergic neurotransmission, this may explained the stimulatory effects of nucleo CMP on critical flicker-fusion frequency which reflects improvement in arousal and sensory function.

Combined effects of lipoic acid and nucleo CMP produced a potential significant effect on critical flicker frequency, critical fusion frequency, fusion percent and fusion index.

This effect may be due to dual action of antioxidant and neurotransmission effects that revealed by lipoic acid and nucleo CMP, but nonsignificant effect on CFFF by either nucleo CMP ,lipoic acid or both may be due to small sample size, duration of therapy or drug dosage might be small also, it is nice to mention that few literature reviews mainly about nucleo CMP made us unable to do comparative results, therefore this article may be a base for future central effects of nucleo CMP.

Moreover, CFFF results are affected by different factors that surely dissimilar in various experimental studies like frequency and duration of stimulating flicker, that correlated positively with CFFF so, low frequency around $10 \mathrm{~Hz}$ reduce CFFF while higher frequency around $100 \mathrm{~Hz}$ augment $\mathrm{CFFF}^{27}$ and most of the previous studies were done on the devices which measure chromatic CFFF ,but in the present study luminated CFFF was measured, since ; chromatic CFFF is lower than luminance CFFF in both patient and normal individual due to differences in temporal informative processing capacity also; luminance CFFF mainly affected by neurodegenerative diseases but, demyelinating disease little affect chromatic CFFF but highly deteriorate with luminance CFFF 28,29,30 this is the most important factor for selection of luminance CFFF in our study. All these factors may explain the variability with other studies. Moreover, our study is hampered by sample size and homogeneity of our enrolled participants, all of our participants were medical college students and this may affected our results so, additional research might evaluate whether older participants would affect our results regarding other high scale CFFF test.

\section{CONCLUSION}

We conclude that lipoic acid and nucleo CMP produced significant arousal effects on normal healthy volunteers and improve cognitive functions.

\section{SUMMARY}

- Lipoic acid improves flicker frequency (descending in normal healthy volunteers)

- Nucleo CMP leads to a significant amelioration of critical flicker frequency, flicker percent and fusion percent.

- Lipoic acid and Nucleo CMP in combination produced significant effects on critical flicker frequency, critical fusion frequency, flicker percent and fusion percent.

- Lipoic acid and nucleo CMP produced improve cognitive functions. 


\title{
ABBREVIATIONS USED
}

\author{
CFFF: Critical fusion flicker frequency.
}

\section{ABOUT AUTHOR}

Hayder M. Al-kuraishy is an Assistant Professor at the Clinical Pharmacology \& Therapeutics Department at Al-Mustansiriya College of Medicine, Baghdad (Iraq). He is positioned as head of research unit and director of postgraduate students in Clinical Pharmacology and Therapeutics Department at Al-Mustansiriya College of Medicine. Dr. Al-kuraishy has an excellent experience in the area of Neuro pharmacology, Pharmacognosy and Natural Products and its influence on psychomotor performance test, Immuno-pharmacology, and antimicrobial drugs.

Ali Ismail Al-Gareeb obtained his Ph.D. degree in 2009 from College of Medicine, Baghdad University (Iraq). Currently, he is Assistant Professor in Clinical Pharmacology and positioned as Head of Clinical Pharmacology \& Therapeutics Department at Al-Mustansyriia College of Medicine, Baghdad (Iraq).

Dr. Al-Gareeb is working on various aspects of cardiovascular, endocrine, and psychomotor related disorders and engaged in finding influence of certain medicine on cardiovascular disease, metabolic disorders and psychomotor performance.

\section{REFERENCES}

1. Simonson E, Brozek J. Flicker fusion frequency; background and applications. Physiol Rev. 1952; 32: 349-78

2. Gandon JM, Allain H. Lack of effect of single and repeated doses of levocetirizine a new antihistamine drug, on cognitive and psychomotor functions in healthy volunteers. Br J ClinPharmacol 2002; 54: 51-8.

3. Seitz AR, Nanez JE, Holloway SR, Watanabe T.Visual experience cansubstantially alter critical flicker fusion thresholds. Hum Psychopharmacol. 2005; 20:55-60

4. Bernardi L, Costa VP, Shiroma LO. Flicker perimetry in healthy subjects:influence of age and gender, learning effect and short-term fluctuation. Arq BrasOftalmol. 2007; 70: 91-99.

5. Petukhov IV, Rozhentsov VV, Aliev MT. On the accuracy of evaluationsof temporal characteristics of visual perception. Bull Exp Biol Med.2007; 144: 267-8.

6. Yen-Yu I. Shih, Lin Wang, Bryan H. De La Garza, Guang Li, et al.Quantitative Retinal and Choroidal Blood Flow During Light, Dark Adaptation and Flicker Light Stimulation in Rats Using Fluorescent Microspheres. Curr Eye Res. 2013; 38(2): 292-298.

7. Jiang T, Yin F, Yao J, Brinton RD, Cadenas E.Lipoic acid restores age-associated impairment of brain energy metabolism through the modulation of Akt/JNK signaling and PGC1 $\alpha$ transcriptional pathway. Aging Cell. 2013;12(6):1021-31.

8. Kumkum Agarwal, Ranjana Varma. Antioxidant Ability of Some Common Indian Vegetables. Journal of Young Pharmacists, 2015; 7(3):262-66

9. Doulames V, Lee S, SheaTB. Environmental enrichment and social interaction improve cognitive function and decrease reactive oxidative species in normal adult mice. Int J Neurosci. 2014; 124(5):369-76.

10. Gulati P, Singh N. Neuroprotective mechanism of ischemic postconditioning in mice: a possible relationship between protein kinase $\mathrm{C}$ and nitric oxide pathways. J Surg Res. 2014 1;189(1):174-83.

11. Soni V, Jain A, Khare P, Gulbake A, Jain SK. Potential approaches for drug delivery to the brain: past, present, and future. Crit Rev Ther Drug Carrier Syst. 2010;27(3):187-236

12. Flierl U, Nero TL, Lim B, Arthur JF, Yao Y, Jung SM, et al. Phosphorothioate backbone modifications of nucleotide-based drugs are potent platelet activators. $J$ Exp Med. 2015;212(2):129-37.

13. Thomann J, Baumann CR, Landolt HP, Werth E. Psychomotor vigilance task demonstrates impaired vigilance in disorders with excessive daytime sleepiness. J Clin Sleep Med. 2014;10(9):1019-24.

14. Fisher BC, Garges DM, Yoon SY, Maguire K, Zipay D. Sex differences and the interaction of age and sleep issues in neuropsychological testing performance across the lifespan in an ADD/ADHD sample from the years 1989 to 2009 . Psychol Rep. 2014;114(2):404-38.

15. Ferrara SD, Zanconer S., Giorge Hi R, low blood alcohol concentration and driving impairment int. J. leg Med. 1994 106: 169-177.

16. Suchy J, Chan A, Shea TB. Dietary supplementation with a combination of alpha-lipoic acid, acetyl-L-carnitine, glycerophosphocoline, docosahexaenoic acid, and phosphatidylserine reduces oxidative damage to murine brain and improves cognitive performance. Nutr Res. 2009;29(1):70-4.

17. Macias-Barragan J, Caligiuri A, García-Banuelos J, Parola M, Pinzani M, Armendariz-Borunda J. Effects of alpha lipoic acid and pirfenidone on liver cells antioxidant modulation against oxidative damage. Rev Med Chil. 2014;142(12):1553-64

18. De Freitas RM. Lipoic Acid increases hippocampal choline acetyltransferase and acetylcholinesterase activities and improvement memory in epileptic rats. Neurochem Res. 2010;35(1):162-70.

19. Cheng B, Zhang Y, Wang A, DongY, Xie Z. Vitamin C Attenuates Isoflurane-Induced Caspase-3 Activation and Cognitive Impairment. MolNeurobiol. 2014 ;4(3):55-67

20. Mulla W, Kuchekar S Thorat V Chopade A Kuchekar B. Antioxidant, Antinociceptive and Anti-inflammatory Activities of Ethanolic Extract of Leaves of Alocasia indica (Schott.). J Young Pharm. 2010; 2(2):137-43.

21. Bersier MG, Miksztowicz V, Peña C, Rodríguez de LoresArnaiz G. Modulation of aspartate release by ascorbic acid and endobain $\mathrm{E}$, an endogenous $\mathrm{Na}^{+}, \mathrm{K}^{+}$ -ATPase inhibitor. Neurochem Res. 2005;30(4):479-86.

22. Mani V, Ramasamy K, Ahmad A, Wahab N. Effects of the total alkaloidal extract of Murraya koenigii leaf on oxidative stress and cholinergic transmission in aged mice. Phytother Res. 2013;27(1):46-53.

23. Mehrotra A, Sandhir R. Mitochondrial cofactors in experimental Huntington's disease: behavioral, biochemical and histological evaluation. Behav Brain Res. 2014;261:345-55.

24. Villinger S, Giller K, Bayrhuber M, Lange A, Griesinger C, Becker S, et al. Nucleotide interactions of the human voltage-dependent anion channel. J Biol Chem. 2014;289(19):13397-406

25. Azran S, Förster D, Danino O, Nadel Y, Reiser G, Fischer B. Highly efficient biocompatible neuroprotectants with dual activity as antioxidants and P2Y receptor agonists. J Med Chem. 2013;56(12):4938-52.

26. Kovács Z, Slézia A, Bali ZK, Kovács P, Dobolyi A, Szikra T, et al. Uridine modulates neuronal activity and inhibits spike-wave discharges of absence epileptic Long Evans and Wistar Albino Glaxo/Rijswijk rats. Brain Res Bull. 2013;97:16-23.

27. Julia Boyle, Philippe Danjou, Robert Alexander, Nicole Calder, Cynthia Gargano, Nancy Agrawal, Irong Fu, Jacqueline B. McCr, et al. Tolerability, pharmacokinetics and night-time effects on postural sway and critical flicker fusion of gaboxadol and zolpidem inelderly subjects. Br J ClinPharmacol. 2009; 67(2): 180-90.

28. Riva CE, Logean E, Falsini B. Temporal dynamics and magnitude of the blood flow response at theoptic disk in normal subjects during functional retinal flicker-stimulation. Neurosci Lett. 2004;356:75-8.

29. Iverson GL, Brooks BL, Ashton Rennison VL. Minimal gender differences on the CNS vital signs computerized neurocognitive battery. ApplNeuropsychol Adult. 2014;21(1):36-42.

30. Shankar $\mathrm{H}$, Pesudovs $\mathrm{k}$. Critical flicker fusion test of potential vision. J Cataract Refract Surg 2007; 33: 232-9. 